Association for Information Systems AIS Electronic Library (AISeL)

$12-31-2002$

\title{
Exploring Consumer Demand for Networked Services: The Importance of Content, Connectivity, and Killer Apps in the Diffusion of Broadband and Mobile Services
}

Catherine Middleton

Ryerson University

\section{Recommended Citation}

Middleton, Catherine, "Exploring Consumer Demand for Networked Services: The Importance of Content, Connectivity, and Killer Apps in the Diffusion of Broadband and Mobile Services" (2002). ICIS 2002 Proceedings. Paper 36.

http://aisel.aisnet.org/icis2002/36 accepted for inclusion in ICIS 2002 Proceedings by an authorized administrator of AIS Electronic Library (AISeL). For more information, please contact elibrary@aisnet.org. 


\title{
EXPLORING CONSUMER DEMAND FOR NETWORKED SERVICES: THE IMPORTANCE OF CONTENT, CONNECTIVITY, AND KILLER APPS IN THE DIFFUSION OF BROADBAND AND MOBILE SERVICES
}

\author{
Catherine A. Middleton \\ School of Information Technology Management \\ Ryerson University \\ Toronto, ON Canada \\ cmiddlet@ryerson.ca
}

\begin{abstract}
What drives consumers to adopt new networked services and technologies? This paper illustrates the differences between content is king and person-to-person communication is the killer app perspectives on what will drive the adoption of mobile technologies. Through an exploration of user and provider understandings of content and interactivity, it is demonstrated that demand for broadcast-type content (e.g., video on demand) is different than demand for connectivity services (e.g., messaging, e-mail). It is noted that content providers are reliant upon finding a content-based killer application to sustain their revenue streams (and thus endorse the content is king perspective), whereas network providers can thrive whether they focus on meeting consumer demand for content or demand for connectivity (or both). Consumer experience with earlier communication networks (e.g., telephone, Internet) demonstrates a strong demand for services that facilitate person-to-person communication.
\end{abstract}

\section{INTRODUCTION}

As noted in the call for papers for the Time, Space and Mobility track for this conference, there has yet to be strong consumer demand for content that is delivered over mobile networks. While NTT DoCoMo's i-mode service has had some success in providing content to mobile users in Japan, much mobile usage is for the purpose of person-to-person communication. Because there is currently little academic research published on the topic of consumer usage of mobile devices, it is difficult to determine the extent to which content might be a driver for the adoption of mobile applications. Many network and content providers believe that in the longer term, content will be king (i.e., the availability of content will be a significant driver for the uptake of mobile services). But the scant evidence that does exist about adoption of mobile devices and networks parallels that of earlier communication technologies, and suggests that the killer application ${ }^{1}$ is connectivity (e.g., person-to-person communication), not content.

The research question guiding this paper is simple. What can be learned about the adoption of mobile technologies from an examination of previous consumer experiences with similar technologies? To address this question, the paper provides a

\footnotetext{
${ }^{1}$ The term killer application, or killer app, is used widely to signify a product or service that will drive demand for, or increase sales of, a related product or service. Searches for compelling applications that legitimize or justify the adoption of particular technologies have been recognized in the computer industry for many years (see Bragitikos 1996; Moore 1994).
} 
framework, developed in the context of the adoption of broadband network services, that explains why consumers appear to be less interested in content and more interested in connectivity. The implications for the adoption of mobile services are outlined. It is suggested that demand for person-to-person connectivity will continue to be a key factor influencing the adoption of mobile networks and devices.

\section{WHAT DRIVES CONSUMER ADOPTION OF NEW TECHNOLOGIES? TWO DISTINCT PERSPECTIVES}

\subsection{Is Person-to-Person Communication the Killer App?}

Most evidence regarding consumer usage of mobile devices comes from the popular press and from consulting reports, meaning that reliable and accessible information is often difficult to obtain. However, what is interesting about anecdotal evidence noting the popularity of person-to-person communication among mobile users is that it is entirely consistent with consumer usage patterns of earlier networked devices. This section provides a brief overview of research findings regarding the adoption of established communication technologies like the telephone, videotex, and the Internet, and shows that consumer adoption of residential broadband networks and mobile devices appears to be occurring in a manner consistent with the adoption of the more established technologies and devices.

Fischer's $(1988,1992)$ studies of how the telephone was adopted show that its initial usage was modelled after the telegraph. As such, it was intended for sending messages. It was also used as a broadcast device, transmitting music and news to telephone subscribers (Aronson 1977; Briggs 1977). Social uses (e.g., chatting with friends and family) were strongly discouraged. It wasn't until the 1920s that the telephone was promoted as a mechanism for sociability (Fischer 1988), yet this is by far the most common usage for consumers today. The telephone is not a one-way transmission device, it is used for person-to-person interaction with friends, family and associates.

In France, the Minitel system quickly moved from a service that provided primarily person-to-machine interaction and communications (e.g., telephone directory listings) to one where person-to-person messaging was popular (Allen 1988). In Canada, it was noted that "successful marketing of videotex [would] be contingent upon the development of interactive transactional services" (Wilson 1984, p. 12). When the Canadian Telidon videotex system did not develop a market for transactional services, it was generally considered a failure. But as Devon (1991) and Slofstra (1984) note, this assessment overlooked an active community of individuals who used the system as a means of communicating and sharing graphics.

Studies of how consumers are using the Internet offer insights into what consumers are doing when they go online. Initial studies considered the use of the Internet by means of dial-up modem (e.g., the HomeNet study, see Kraut et al. 1999; Kraut et al. 1996). The HomeNet researchers concluded:

Despite the newness and excitement surrounding the World Wide Web, the killer application on the Internet is still interpersonal communication. Electronic mail use is more popular than use of the Web, more stable, and drives greater and more lasting use of the Internet overall (HomeNet 2000).

Nie and Erbring's (2000) research (based on a sample of more than 4,000 Americans) found that e-mail was the most common Internet activity; 90 percent of those who used the Internet used e-mail. More recent studies by the UCLA Center for Communication Policy $(2000,2001)$ confirm that e-mail is widely used by Americans. For example, experienced users (with 5 or more years of Internet use) spend about a quarter of their time online on e-mail, in comparison to less than 3 percent of their time downloading music or playing games. When Jupiter MediaMetrix assessed AOL's services in early 2001, it found that more than 60 percent of users' online time was spent on messaging or e-mail, with a total of 9 billion minutes spent on instant messaging alone (reported by National Research Council 2002).

Many consumers in wired nations around the world are now shifting from dial-up Internet access to broadband. Although many governments are interested in extending broadband network access to all of their citizens (Directorate for Science Technology and Industry 2001; National Broadband Task Force 2001; National Research Council 2002), there is still very little academic research that considers how consumers will adopt broadband technologies. (The recent National Research Council study on broadband usage in the United States gathered information from magazines, newspapers, practitioner journals, vendors' white papers, and consultants.) Some research has been conducted on consumer responses to a trial of broadband services, but has yet to be widely disseminated (Hampton 2001; Middleton 2002a, 2002b). What this research suggests, however, is consistent with 
user behaviors outlined above. Even when provided with free access to services like videophones and music on demand, users spent most of their time online engaged in person-to-person communication, either via e-mail or by interacting with other trial members via a community mailing list.

There is even less academic research about usage of mobile devices. The evidence presented here is drawn from secondary sources, which is problematic because it is not always possible to determine the quality of the data collection or analysis processes. What is of interest, though, is not precise reports of consumer behaviors, but the overall concurrence by analysts that consumers are heavy users of mobile person-to-person communication services. For example, a recent study by IDC Malaysia suggests that 88 percent of subscribers were using short messaging service (SMS) on their mobile devices, compared to only 5 percent who were browsing Web sites using wireless application protocol (WAP) (Mok 2002). The conclusion that SMS is extremely popular among mobile phone users is supported by Frengle (2002), who notes that in Japan, mobile phone users between the ages of 19 and 29 used text messaging about 68 percent of the time. Of all Internet-enabled mobile phone users in Japan, just over a quarter (26.1\%) use their phones to surf the Internet. In addition, NTT's DoCoMo i-mode service is being used for "mail in the majority of cases" (Frengle 2002, p. 82). Most of its revenue comes from telephone services, despite the fact that there are more than 1,600 official content sites available to DoCoMo's i-mode subscribers.

It is too early to tell whether the usage of mobile devices will continue to be dominated by person-to-person communication applications like e-mail and messaging, but based on adoption patterns for other communication technologies, this outcome would not be at all surprising. Over time, academic research will address this issue with detailed studies of large samples of mobile users. But until then, it is useful to consider past experiences as possible harbingers of future diffusion of mobile technologies and devices.

\subsection{Content is King-Or Is It?}

The section above has detailed one perspective on what might drive the wider-scale diffusion of mobile devices in future years. But there is a second perspective, which is perhaps best described by the phrase "content is king." According to this viewpoint on the diffusion of new technologies, content is the killer application for which consumers (and network providers) are waiting. Content is what will drive consumers to adopt new communication services. There is a persistent and widely-held belief among broadband and mobile network and infrastructure providers that content-based killer applications will be found, even though such applications are not evident today (Heinzl 2001; Kupfer 2000). Consistent with this viewpoint is the idea that without content, network infrastructure is of little value. The following quotations offer examples of this thinking, as applied to the development of broadband networks:

If the content is not attractive and convenient, if the price is not right, and if the system is not robust - it will not succeed (Zahariadis et al. 1997, p. 63).

Without interesting and stimulating content, interactive multimedia will not succeed in the long term (Di Concetto et al. 1999, p. 44).

Content is what consumers want to see. They want to see a value proposition. And access alone is not a value proposition. Or it's a value proposition that can't be sustained. After a while, consumers want more than just access (Participant in Broadband Trial, Interviewed in 1999).

Applications are going to be the regulator and demand creator for broadband... Broadband is never going to take off without them....Customers are looking for the application that makes the broadband world touchable and believable to them, that underscores its benefit. And that application does not yet exist (Ford Cavallari, Internet Consultant, in Zerega 2000, p. 111).

I don't want to be anyone's dumb pipes....If all you do is racks and servers, that's dumb. What we're doing is melding the network and the content (Leo Hindery, CEO, Global Crossing, in Krause 2000).

I always thought that music revitalized our audio hardware business, and that buying a studio would strengthen our TV business. The same is true for broadband: Without content, the network is nothing (Norio Ogha, Sony Corporation Chairman, in Schlender 2000). 
Some broadband providers are trying to make the technology more enticing to the masses. That involves making broadband "content," a catchall term referring to anything and everything that gets zapped over broadband lines, more appealing to ordinary subscribers (Cauley 2001).

Telecom carriers are on the hunt for broadband "killer apps" to fill up their fat, often largely empty pipes (Heinzl 2001).

To fully justify fibre to the home, we need very high bandwidth services that are not here today (Huppé 2001).

As Carlyle (2002) observes, "the critical application which will make everyone desperate to use high bandwidth services—and pay for them—-has proved elusive." Writing about mobile devices, another British commentator notes:

The main reason users like me are looking forward to $3 \mathrm{G}$ is as a way of getting broadband connectivity from anywhere. What we're after is a way of keeping in touch with one another, not with CNN, HSBC, Nasdaq nor even, God help us, Arsenal.com. What we want is a way of connecting our laptops to fast, reliable, always-on, ubiquitous email. And therein lies the Telco problem: there is no money to be made from email because the user owns the content (shock, horror!) so there is no way of raking off a percentage from content providers (Naughton 2001).

Despite the skeptical comments, it is too early to conclude that the content is king model is not favored by mobile consumers. However, it does appear that content-based applications have yet to gain widespread consumer acceptance, meaning that they are not currently drivers of demand for mobile services.

\section{HOW AN UNDERSTANDING OF INTERACTIVITY HELPS EXPLAIN THE ADOPTION OF BROADBAND AND MOBILE SERVICES}

This paper argues that in order to understand the deployment of mobile devices, it is instructive to learn from consumer experiences with earlier communication technologies. In the section that follows, a model (developed by the author in the context of residential broadband services) shows how the content is king perspective differs from the one that suggests that the killer application is person-to-person connectivity. It is demonstrated that the two perspectives are centered on fundamentally different assumptions about how content and interactivity are defined, and about what makes content valuable for consumers and providers. $^{2}$ In particular, it is noted that the ability to control both the communication process and content generation and distribution is highly valued by consumers.

Communication networks are designed to support interactivity. But a review of the literature (see Middleton 2002a) shows that there is no consensus as to how interactivity is defined (Jensen 1998; Rafaeli 1988). Multiple authors do, however, recognize that the concept encompasses two dimensions, process and content, which are described below.

\subsection{Control over the Communication Process}

Interactive communication is distinct from mass media broadcasting because it enables recipients to influence how the communication process occurs. When communication is interactive, users can manage their exchanges with content providers. This is in contrast to a broadcast situation (where the content provider determines when information is sent over the communication network, and users have no mechanism by which to communicate directly with the content provider to influence this process). To allow input into the communication process, users must be able to send and receive messages using the available communication medium. Although media that allow for two-way messaging can be used in non-interactive ways, media that only allow for unidirectional communication cannot support interactivity.

\footnotetext{
${ }^{2}$ Unless otherwise noted, the term provider refers here to network providers (e.g., telcos, cablecos, Internet service providers) and content providers (e.g., entertainment companies, artists). The terms user and consumer are used interchangeably to refer to people accessing the services offered by the providers.
} 
Rogers (1995) describes interactivity as "the degree to which participants in a communication process can exchange roles in and have control over their mutual discourse" (p. 314). This definition emphasizes the importance of controlling the communication process, and also introduces a second dimension, the equivalence of sender and receiver, which is discussed below.

\subsection{Control of Content}

Consistent with Rogers' position, McMillan and Downes (2000) observe that "in interactive communication, the roles become interchangeable" (p. 163). In an interactive context, then, all parties to an information exchange are peers. This means that anyone who is connected to a network can provide information (content) to any other networked user. This is in marked contrast to a broadcast model where only the broadcaster can determine what content is distributed. Heeter (1989) refers to this aspect of interactivity as the ease of adding information, and defines it as "the degree to which users can add information to the system that a mass undifferentiated audience can access" (p. 224).

\subsection{A Model of Interactivity}

As outlined above, the two dimensions on which interactive and mass communication can be differentiated are: (1) control of the communication process and (2) control of the content. Depending on who controls each dimension (i.e., provider or user), a communication service exhibits the characteristics of one of four levels of interactivity. The four levels of interactivity are described using a two by two matrix model, ${ }^{3}$ which is illustrated in Figure 1. The model explores the assumptions about control of content and process that are implicit in the content is king and the killer app is connectivity perspectives outlined earlier. It is demonstrated that the person-to-person communication services apparently favored by consumers are ones that exhibit full interactivity. In contrast, content-based services, as envisaged by content providers, do not allow users control over the creation or sharing of content.

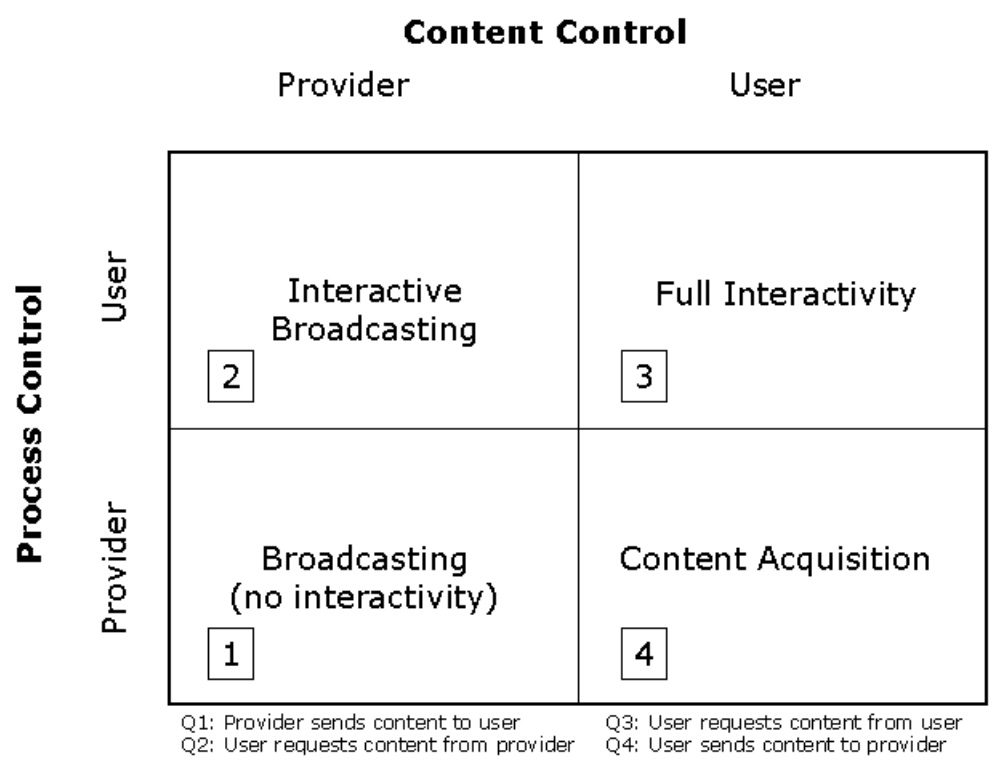

Figure 1. A Two-Dimensional Model of Interactivity

\footnotetext{
${ }^{3}$ There are some similarities between this model and a model proposed by Bordewijk and van Kaam (1986) to demonstrate how new communication technologies empower some groups while disenfranchising others. The Bordewijk and van Kaam model is not used to explore the meaning of interactivity, nor the adoption of broadband or wireless services.
} 
Figure 1 offers terminology that can be used to describe each quadrant in the matrix. The level of interactivity ranges from none in quadrant 1 (broadcasting) to full interactivity in quadrant 3, with partially interactive services in quadrants 2 and 4 . In order for communication to be fully interactive, a user must be able to control the communication process and to generate content or information for exchange with other users. As quadrant 1 shows, a situation in which the provider controls both the communication process and the content being communicated is not an interactive situation, but a broadcast one. In quadrant 2 , the user has control over the communication process, but has no input into the content that is communicated, hence it is described as interactive broadcasting. In quadrant 4, content acquisition, the provider controls the communication process, but the user generates content (provider surveillance of user actions is an example of a quadrant 4 activity). Both quadrants 1 and 4 describe media usage where control of the communication process rests with the service provider. Providers' ability to maintain tight control over user media experiences is being steadily eroded as consumers embrace interactivity. In fact, the two perspectives on consumer adoption of broadband (and other) networks and devices can be mapped onto quadrants 2 and 3 , thus these quadrants are the focus of this discussion.

In quadrant 2 , content is generated and owned by content providers. Providers can offer value to users by enabling them to choose how and when they will engage this content without giving up ownership of the content. The quadrant is the home of high bandwidth services such as video and music on demand and interactive television. Lower bandwidth services include customer service applications such as online banking, shopping, or computing support (e.g., querying databases to find answers to questions, downloading files). In this quadrant, content is king.

In quadrant 3, no single provider controls content. In addition, because users can determine how and when they receive content, in this quadrant communication is fully interactive. The term service is less applicable here than elsewhere. Users do need someone to provide them with network access, but that is the only service that users require. Content, provided by broadcasters in quadrants 1 and 2, can be provided by any user in quadrant 3, as users are peers on the network. All users have full access to send and receive content in any form. This is where peer-to-peer networking and person-to-person communication flourish.

Popular applications include e-mail, community listservs, news groups, instant messaging and SMS. Higher bandwidth, fully interactive applications have also been in demand in the past few years. Perhaps the best-known is the now defunct Napster, which provided a mechanism for people to search for music files and download them from other Napster users' computers. Napster is a good example of a service that empowers users, allowing them full control over communication processes and content. What is particularly interesting about Napster, however, is how it has influenced consumers' understanding of ownership of content. Many consumers now believe that they should have the right to copy and distribute music, and other content, without limitations imposed upon them by the content creators or the courts. This belief is reflected in the many content exchange programs, inspired by Napster, encompassing not just music, but video as well (e.g., Aimster, DivX, Fasttrack, Gnutella, Internet Relay Chat, Morpheus). Regardless of whether consumers are sharing content over which they have copyright, or whether they are sharing content that does not belong to them, demand for fully interactive services is expected to continue its growth.

\section{DISCUSSION}

How can the conceptual framework developed here be used to understand the adoption of new communication technologies and devices? Its value comes from a recognition of the dual components of interactivity. Interactivity is frequently defined as the extent to which the communication process is controlled by the user or provider. What this model demonstrates is that it is differences in understandings of who controls content that influence perspectives on the diffusion of new communication technologies.

In the past, network providers (e.g., cable companies) had complete control over content that was broadcast to consumers (i.e., they operated in quadrant 1). To meet perceived consumer demands for interactivity, services like video on demand and interactive television were proposed. This represented a shift (for providers) from quadrant 1 to quadrant 2 (see Figure 2). As has been noted, it is in quadrant 2 where content is king. In quadrant 2, consumer demand for networks is driven by the content that can be delivered over the networks. (It is also noted that the content is king model, in the context of broadband services, is dependent upon the availability of high bandwidth network access for consumers, as represented in the upper triangle in quadrant 2.) According to this perspective, without content, network access is of little value to consumers.

The framework set forth here cannot predict demand for networked devices and services, but it does illustrate the components of such demand. Thus, while there is likely to be a market for interactive broadcast services (like video on demand), the provision of such services does not help meet the demonstrated consumer demand for more control over content (as illustrated in quadrant 3 ). Interacting with friends and communities of interest is a different activity than consuming broadcast content (whether 


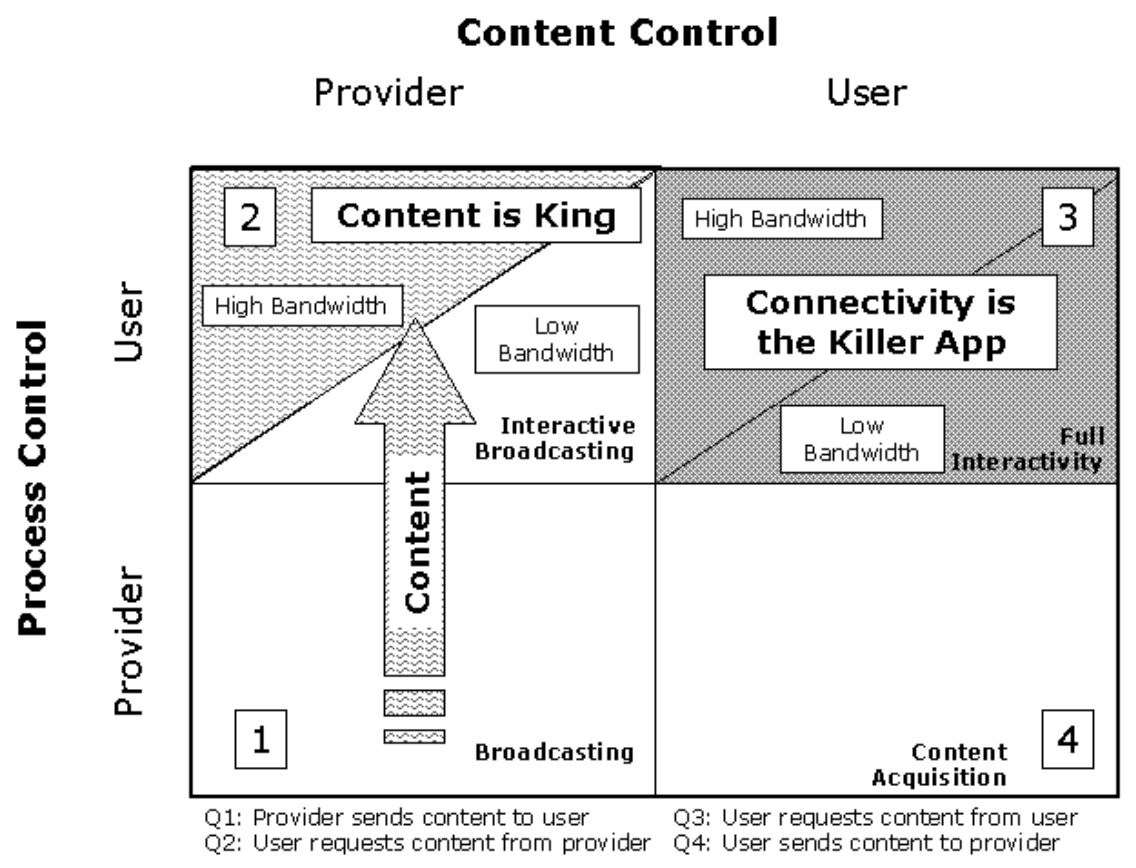

Figure 2. Revised Model showing Content is King and Connectivity is the Killer App Perspectives

it is interactive or not). What the diffusion and adoption of the telephone and low bandwidth Internet access suggest is that consumers prefer to use communication networks for connecting with other people (i.e., quadrant 3), rather than for receiving content (quadrant 2).

While consumers appear to favor quadrant 3, the model presented here suggests that it will always be more beneficial for content providers to encourage the adoption of services that are in quadrant 2 than those that are in quadrant 3 . This is because content providers derive their revenues from selling content. Interestingly, network providers can thrive in either quadrant 2 or 3 , but many network providers have aligned themselves with content providers (through mergers or strategic alliances), believing that demand for their network services would be driven by the availability of content. It is not clear that network providers fully understand that consumers find value in creating their own content. If network providers did understand this, they could develop new services that would help users interact with each other.

\section{IMPLICATIONS FOR THE DIFFUSION OF MOBILE DEVICES}

The framework presented above was derived from data relating to consumer adoption of residential broadband networks, but the insights it generates can inform research on the adoption of mobile devices (and other new communication technologies). In particular, the differentiation between the content is king and connectivity is the killer app perspectives is a key insight for anyone wishing to understand the diffusion of any type of content delivery networks and associated content. The model demonstrates that consumer demand for content is influenced by the extent to which the consumer can control or generate content, and that broadcast-type content meets different needs (primarily entertainment-based) than does fully interactive content (which provides interpersonal connectivity). As such, it is not surprising that some stakeholders in the mobile industry are promoting content-based killer applications as a means of stimulating demand for network access, whereas consumers are already showing that demand for connectivity (e.g., SMS and e-mail) is present with any type of communication network.

Assuming that consumers will continue to demand services that exhibit full interactivity, the following strategies are suggested for service providers: ${ }^{4}$

\footnotetext{
${ }^{4} \mathrm{~A}$ more detailed discussion of suggested strategies is provided in Middleton (2002a).
} 
- network providers can shift their focus from obtaining third party content (and developing alliances with content providers) to offering tools that allow consumers to better develop and manage their own content (e.g., search and edit tools)

- network providers can improve infrastructure to allow for easier peer-to-peer interaction (e.g., symmetrical networks)

- content providers can attempt to develop content that is fully interactive, perhaps by building community spaces that make it easier for users to interact with each other, or by developing multi-player games that allow peer-to-peer interactivity.

\section{FURTHER WORK}

This paper presented two perspectives on what drives the adoption of mobile technologies as distinct entities, but it is noted that the extent to which users can control content is not an all or nothing proposition. In addition to generating their own content (e.g., messages), consumers can get some measure of control over their communication activities by personalizing their own interfaces. This observation may explain the popularity of downloadable ring tones, one example of the ways in which mobile users can customize their own devices. It is also noted that there are other Web-based applications (e.g., banking) that are not easily categorized as content- or connectivity-based services. The positioning and importance of such utility or transaction type applications is not considered in this paper, but should be incorporated as part of future research in this area.

There is much to be learned about the adoption of mobile technologies. As this paper demonstrates, past experience with new communication technologies does offer insights as to how the diffusion of consumer mobile devices may unfold. It is now time to conduct detailed studies on consumer attitudes toward content and connectivity services provided by mobile devices. These sorts of comprehensive studies will collect essential data that can be used to test the conclusions of this paper. It will also be instructive to look at user experiences in areas like Scandinavia, where mobile devices have already achieved very high penetration rates, and to assess Japanese experiences with services like i-mode.

\section{REFERENCES}

Allen, D. "New Telecommunication Services: Network Externalities and Critical Mass," Telecommunications Policy (12:3), 1988, pp. 257-271.

Aronson, S. H. "Bell's Electrical Toy: What's the Use? The Sociology of Early Telephone Usage," in I. de Sola Pool (ed.), The Social Impact of the Telephone. Cambridge, MA: MIT Press, 1977, pp. 15-39.

Bordewijk, J. L., and van Kaam, B. “Towards a New Classification of Tele-Information Services,” Intermedia (14:1), 1986, pp. $16-21$.

Bragitikos, M. "Just What Will Take the Net from a Novelty to a Necessity?," Wall Street Journal Interactive Edition, 6 December, 1996.

Briggs, A. "The Pleasure Telephone: A Chapter in the Prehistory of the Media," in I. de Sola Pool (ed.), The Social Impact of the Telephone. Cambridge, MA: MIT Press, 1977, pp. 40-65.

Carlyle, P. "Content Is King in Battle to Find Killer Application," The Scotsman, 28 March, 2002, p. 5.

Cauley, L. "Up to Speed: Getting High-Speed Internet Access Has Been a Nightmare; Phone and Cable Firms Are Scrambling to Fix That," Wall Street Journal, 12 February, 2001, p. R24.

Devon, T. "Interactivity and the Popular Support for Telidon," Canadian Journal of Communication (16:2), 1991, pp. 307-311.

Di Concetto, M., Pavarani, G., Rosa, C., and Rossi, F. "AMUSE: Advanced Broadband Services Trials for Residential Users," IEEE Network (13:2), 1999, pp. 37-45.

Directorate for Science Technology and Industry. The Development of Broadband Access in OECD Countries. Organisation for Economic Co-operation and Development (OECD), Paris, 2001.

Fischer, C. S. America Calling: A Social History of the Telephone to 1940. Berkeley, CA: University of California Press, Berkeley, 1992.

Fischer, C. S. "Touch Someone: The Telephone Industry Discovers Sociability," Technology and Culture (29), 1988, pp. 32-61. Frengle, N. i-mode: A Primer. New York: Hungry Minds, 2002.

Hampton, K. N. Living the Wired Life in the Wired Suburb: Netville, Glocalization and Civil Society. Unpublished Doctoral Dissertation, University of Toronto, 2001. 
Heeter, C. "Implications of New Interactive Technologies for Conceptualizing Communication," in J. L. Salvaggio and J. Bryant (eds.), Media Use in the Information Age: Emerging Patterns of Adoption and Consumer Use. Hillsdale, NJ: Lawrence Erlbaum Associates, 1989, pp. 217-235.

Heinzl, M. "Broadband Carriers Are Hunting for 'Killer Apps'," Wall Street Journal, June 14, 2001, p. B10.

HomeNet. "The HomeNet Project," Carnegie Mellon University, 2000 (available online at homenet.andrew.cmu.edu/progress, accessed November 5, 2000).

Huppé, J. "Challenges in Pushing Fibre to the Home," presentation to CANARIE Advanced Networks Workshop, Toronto, 2001.

Jensen, J. F. "Interactivity: Tracing a New Concept in Media and Communication Studies,” Nordicom Review (19:1), 1998, pp. 185-204.

Krause, J. "Global Crossing Plans Its Media Play: New CEO Leo Hindery Is Hunting for Content to Fill the Company's Vaunted Worldwide Network," Industry Standard, 3 April, 2000.

Kraut, R., Mukhopadhyay, T., Szczypula, J., Kiesler, S., and Scherlis, B. "Information and Communication: Alternative Uses of the Internet in Households," Information Systems Research (10:4), 1999, pp. 287-303.

Kraut, R., Scherlis, W., Mukhodaphyay, T., Manning, J., and Kiesler, S. "The HomeNet Field Trial of Residential Internet Services," Communications of the ACM (39:12), 1996, pp. 55-63.

Kupfer, A. "Broadband: Still a Killer App Away," Fortune, February 7, 2000, pp. 27-28.

McMillan, S. J., and Downes, E. J. "Defining Interactivity: A Qualitative Identification of Key Dimensions," New Media \& Society (2:2), 2000, pp. 157-179.

Middleton, C. A. "Content and Connectivity: Competing Perspectives on Success in a Residential Broadband Network Trial," Unpublished Doctoral Dissertation, York University, 2002a.

Middleton, C. A. "Learning from the Netcom Trial: Implications for the Deployment of Residential Broadband Networks," in M. E. Hurt (ed.), Proceedings of the Annual Conference of the Administrative Studies Association of Canada. Winnipeg, MB, Canada: ASAC Information Systems Division, 2002b, pp. 70-80.

Mok, M. "Popular Mobile Data Services," New Straits Times, May 1, 2002, p. 22.

Moore, S. "The Killer App Crew," Computerworld, May 23, 1994, pp. 145-146.

National Broadband Task Force. The New National Dream: Networking the Nation for Broadband Access. Ottawa: Industry Canada, 2001.

National Research Council. Broadband: Bringing Home the Bits. Washington, DC: National Academy Press, 2002.

Naughton, J. "The Networker: Why the 3 G Gamble Looks a Busted Flush," The Observer, July 8, 2001, p. 7.

Nie, N. H., and Erbring, L. Internet and Society: A Preliminary Report. Stanford, CA: SIQSS, 2000.

Rafaeli, S. "Interactivity: From New Media to Communication," in R. P. Hawkins, J. M. Wiemann, and S. Pingree (eds.), Advancing Communication Science: Merging Mass and Interpersonal Process. Newbury Park, CA: Sage Publications, 1988, pp. 110-134.

Rogers, E. M. Diffusion of Innovations(4th ed.). New York: The Free Press, 1995.

Schlender, B. "Sony Plays to Win," Fortune (141:9), May 1, 2000, pp. 142-157.

Slofstra, M. "User Group Speaker Tries to Dispel Myths About Telidon," Computing Canada (10:11), 1984, p. 14.

UCLA Center for Communication Policy. The UCLA Internet Report-Surveying the Digital Future. Los Angeles: Regents of the University of California, 2000.

UCLA Center for Communication Policy. The UCLA Internet Report 2001 - Surveying the Digital Future. Los Angeles: Regents of the University of California, Los Angeles, 2001.

Wilson, K. "The Wired Home: An Assessment of Market and Ownership Trends in the Videotex Industry and Their Import for Public Policy," McGill University Graduate Program in Communications, 1984.

Zahariadis, T., Rosa, C., Pellegrinato, M., Lund, A. B., and Stassinopoulos, G. "Interactive Multimedia Services to Residential Users," IEEE Communications Magazine (35:6), 1997, pp. 61-68.

Zerega, B. "Highway to Nowhere," Forbes ASAP, February 21, 2000, pp. 111-112. 